\title{
The Correlation between Childhood Hydrocephalus and Bridging Cortical Vein Cross-sectional Area: Is there an Underlying Impedance Pump Malfunction in Hydrocephalus?
}

Grant Alexander Bateman ( $\nabla$ grant.bateman@health.nsw.gov.au )

John Hunter Hospital

Alexander Robert Bateman

University of New South Wales

Gopinath Musuwadi Subramanian

John Hunter Hospital

\section{Research Article}

Keywords: obstructive, communicating, hydrocephalus, cerebral blood flow

Posted Date: February 4th, 2022

DOI: https://doi.org/10.21203/rs.3.rs-1293405/v1

License: (c) (1) This work is licensed under a Creative Commons Attribution 4.0 International License.

Read Full License 


\section{Abstract}

Dogs with a naturally occurring form of hydrocephalus have an elevated transmural venous pressure leading to cortical vein dilatation. The purpose of this study is to discover if there is vein dilatation in childhood hydrocephalus and to estimate the pressure required to maintain any enlargement found. Children with hydrocephalus between the ages of 4 and 15 years were compared with a control group. Magnetic resonance venography (MRV) and flow quantification were performed. The arterial inflow, sagittal sinus and straight sinus venous outflow were measured and the outflow percentages compared to the inflow were calculated. The cross-sectional area of the veins were measured. There were a total of 18 children with hydrocephalus, compared to 72 age and sex matched control MRV's and 22 control flow quantification studies. In hydrocephalus, the sagittal sinus venous return was reduced by $12.9 \%$, but the straight sinus flow was not significantly different. The superficial territory veins were $22 \%$ larger than the controls but the vein of Galen was unchanged. There is evidence of a significant increase in the superficial vein transmural pressure in childhood hydrocephalus estimated to be approximately $4 \mathrm{mmHg}$. An impedance pump model is suggested to explain these findings.

\section{Introduction}

In hydrocephalus, there is deformation of the brain parenchyma. In engineering terms such a deformation requires the expenditure of energy. In a hydraulic system, energy expenditure requires a pressure differential ${ }^{1}$. The pressure difference deforming the brain in hydrocephalus has remained elusive. In a study of adult hydrocephalus, a prediction of an elevation in superior sagittal sinus (SSS) pressure of 3-4 $\mathrm{mmHg}$ above normal was made based on the evidence of increased collateral flow bypassing the sinuses

2. It was hypothesised that collapse of the sagittal sinus would set up a pressure differential between the superficial and deep brain parenchyma and account for the ventricular dilatation found ${ }^{1}$. However, when this differential was estimated using Poiseuille's equation, it was less than predicted being only 1.2 $\mathrm{mmHg}{ }^{1}$.

Another problem with the sinus pressure differential hypothesis arises because of the subarachnoid course of the bridging cortical veins (BCV) and the vein of Galen. The capillary and venular pressure in the brain is thought to be maintained by a Starling resistor mechanism because the veins pass through the subarachnoid space ${ }^{3}$. The cortical veins are a series of collapsible tubes in which the pressure external to the tubes (CSF pressure) usually exceeds the outflow pressure (sinus pressure) therefore the perfusion pressure will only depend on the inflow pressure and the CSF pressure. If the CSF pressure is increased, the pressure in the cortical veins must be slightly higher for blood to flow. Thus, the pressure across the cortical vein wall (transmural pressure) is expected to remain very nearly constant ${ }^{4}$. Therefore, a pressure difference in the sinuses would be irrelevant to the brain parenchyma if both brain territories had their pressures set by the intracranial pressure ${ }^{1}$ and not the outflow sinus pressure.

However, the Starling resistor model may be incomplete in hydrocephalus. Comparing pressures in normal dogs to those with a naturally occurring form of hydrocephalus, the lateral ventricle, cortical vein 
and sagittal sinus pressures in the controls were $10.2,11.7$ and $5.2 \mathrm{mmHg}$ respectively, with hydrocephalic dogs being 15.1, 21.5 and $8.4 \mathrm{mmHg}$ respectively ${ }^{5}$ (see Fig. 1a-b). Note the sagittal sinus pressure is mildly increased by $3.2 \mathrm{mmHg}$ but the BCV pressure is increased by $9.8 \mathrm{mmHg}$ in hydrocephalus compared to controls. The increase in sinus pressure in hydrocephalic dogs can be explained by venous collapse. Modelling of hydrocephalus in dogs shows the entire sinus length to be narrowed ${ }^{6}$ (see Fig. $1 \mathrm{c}-\mathrm{d}$ ). In dogs, between the cortical veins and the superior sagittal sinus, lie the lateral lacunae, which are small venous lakes between the leaves of dura which are thought to act as Starling resistors ${ }^{5}$. The BCV transmural pressure is increased by $327 \%$ in hydrocephalic dogs ${ }^{5}$. The cause of this has never been satisfactorily explained by the Starling resistor mechanism. This suggests that the Starling resistor model is incomplete in canine hydrocephalus. Such a large increase in transmural pressure should be visible as an increase in BCV size (see Fig. 1e-f).

In a recent paper studying children with hydrocephalus, in those between 4-15 years of age there were mild stenoses of the sagittal sinus, the transverse sinuses and the sigmoid sinuses of $35 \%, 30 \%$ and $41 \%$ respectively compared to controls ${ }^{7}$. These stenoses should mildly increase the sinus pressures, similar to the findings in the dogs as already discussed.The purpose of the current study is to 1) estimate the superficial and deep venous territory pressure difference based on blood flow and collateral flow in children and 2) to measure the cross-sectional area of the BCVs at the superficial sinus junction and vein of Galen at the deep junction to see if the predicted pressure difference resides within the BCVs.

\section{Results}

The mean blood flow and sinus size findings are summarized in Table 1. The raw data is appended online in supplementary table $\mathrm{S} 1$. 
Table 1

BLOOD FLOW AND VEIN SIZE

\begin{tabular}{|c|c|c|c|c|c|c|c|c|}
\hline & & Control & $\begin{array}{l}\text { Blood } \\
\text { flow }\end{array}$ & & & & & \\
\hline & Age & Arterial inflow & $\begin{array}{l}\text { SSS } \\
\text { outflow }\end{array}$ & $\begin{array}{l}\text { ST } \\
\text { outflow }\end{array}$ & $\begin{array}{l}\text { SSS \% } \\
\text { return }\end{array}$ & $\begin{array}{l}\mathrm{ST} \% \\
\text { return }\end{array}$ & & \\
\hline & & иог & $\mathrm{ml} / \mathrm{min}$ & $\mathrm{ml} / \mathrm{min}$ & $\%$ & $\mathrm{ml} / \mathrm{min}$ & & \\
\hline mean & 9.2 & 996 & 558 & 162 & 56.5 & 16.2 & & \\
\hline SD & 4.1 & 216 & 132 & 60 & 8.2 & 4.3 & & \\
\hline \multirow[t]{4}{*}{$n$} & 22 & & & & & & & \\
\hline & & Control & $\begin{array}{l}\text { Vein } \\
\text { size }\end{array}$ & & & & & \\
\hline & & & & & & & $\begin{array}{l}\text { BCV } \\
\text { area }\end{array}$ & $\begin{array}{l}\text { Galen } \\
\text { area }\end{array}$ \\
\hline & & & & & & & $\mathrm{mm} 2$ & $\mathrm{~mm} 2$ \\
\hline mean & 9.6 & & & & & & 8.5 & 11.9 \\
\hline SD & 3.3 & & & & & & 2.7 & 4.9 \\
\hline \multirow[t]{2}{*}{$\mathrm{n}$} & 72 & & & & & & & \\
\hline & & Hydrocephalus & & & & & & \\
\hline mean & 9.6 & 1056 & 474 & 144 & 43.6 & 14.0 & 10.4 & 11.2 \\
\hline SD & 3.3 & 336 & 240 & 42 & 15.1 & 3.5 & 3.3 & 3.9 \\
\hline $\mathrm{n}$ & 18 & & & & & & & \\
\hline MWU & 0.76 & 0.70 & 0.06 & 0.34 & $0.003^{*}$ & 0.09 & $0.0008^{*}$ & 0.60 \\
\hline
\end{tabular}

In the controls, as expected there were moderate to strong positive correlations between arterial inflow and both sagittal sinus $(r=0.73, p=0.0001)$ and straight sinus outflow $(r=0.68, p=.0009)$. All other correlations were weak or nonexistent.

In the hydrocephalus patients, there was no significant difference between communicating or noncommunicating or between active and compensated hydrocephalus for any metric. There was no significant correlation between the ventricular size or the aqueduct flow rate for the communicating hydrocephalus patients with any other metric acquired. Comparing hydrocephalus with controls, the sagittal sinus venous return was reduced by $12.9 \%(p=0.003)$. Comparing the 288 control BCVs with the 
72 hydrocephalic patient BCVs sampled, showed the mean bridging cortical vein area was increased by $22 \%(p=0.0008)$. Similar to the controls, there were moderate to strong correlations between the arterial inflow and the sagittal sinus $(r=0.67, p=0.002)$ and straight sinus outflow $(r=0.76, p=0.0002)$. There was a moderate negative correlation between arterial inflow and straight sinus percentage return $(r=-0.64$, $p=0.004$ ) but not the sagittal sinus percentage return. There was a moderate positive correlation between the sagittal sinus flow and the bridging cortical vein area $(r=0.65, p=0.004)$ (see Fig. $2 a)$ and between the sagittal sinus percentage return and bridging vein area $(r=0.67, p=0.002)$ (see Fig. $2 b)$.

\section{Modelling}

In human cadavers the mean diameter of the bridging veins entering the sinus at the vertex is $3.1 \mathrm{~mm}$ and at angiography at the same site it is $3.3 \mathrm{~mm}^{8}$. Which gives a stress free radius (cadavers) of 1.55 $\mathrm{mm}$ and a stress free area of $7.55 \mathrm{~mm}^{2}$. The area with a normal transmural pressure (angiography) would be $8.55 \mathrm{~mm}^{2}$. The mean thickness of the human bridging veins is $0.044 \mathrm{~mm}^{9}$. The normal cortical vein transmural pressure for primates is $2.8 \mathrm{mmHg}{ }^{10}$. Placing these values into equation 1 gives a circumferential Young's modulus of $0.163 \mathrm{MPa}$. Given the normal stressed state area of the BCV's from the present study is $8.5 \mathrm{~mm}^{2}$ and the above parameters for wall thickness and elastic modulus, we can derive the stress free radius for the present study to be $1.55 \mathrm{~mm}$ from equation 1 . Given the stressed state BCV area for the hydrocephalus patients is $10.4 \mathrm{~mm}^{2}$ in this study, the transmural pressure from equation 1 would be $6.9 \mathrm{mmHg}$ or $4.1 \mathrm{mmHg}$ higher than normal.

\section{Discussion}

The hypothesis made in this paper is that the pressure difference required to drive ventricular enlargement in hydrocephalus resides predominately within the bridging cortical veins. In humans, on average 18 cortical veins drain directly into the sagittal sinus without lateral lacunae ${ }^{11}$. The bridging vein diameter remains constant along its subarachnoid course but dilates at a short section called the outflow cuff segment as it joins the sinus ${ }^{11}$. The collagen fibres are helical at this junction and longitudinal elsewhere ${ }^{11}$. In humans the bridging cortical veins are suggested to be passive Starling resistors as previously described. Piechnik et al. predicted that the trans-mural cortical venous pressure change from hyperemia to severe ischemia should only be in the order of $1.5 \mathrm{mmHg}$, with the physiological range being considerably less ${ }^{12}$. This appears to be incorrect. In normal dogs, the cortical vein transmural pressure is $1.6 \mathrm{mmHg}$. This increases by $12.5 \%$ when mock CSF is infused, by $63 \%$ from inhalation of carbon dioxide and by $81 \%$ by jugular vein compression, with all these changes giving an increase in ICP of approximately $10 \mathrm{mmHg}^{5}$. In hydrocephalic dogs the ICP rises by $4.9 \mathrm{mmHg}$ but the transmural pressure increases by $327 \%$. In human subjects, the bridging veins show a mean diameter of $2.04 \mathrm{~mm}$ under normal intracranial pressure and increase to $2.65 \mathrm{~mm}$ under an increased ICP ${ }^{13}$, a $69 \%$ increase in cross-sectional area. Thus, the purpose of the current study is to investigate whether BCV dilatation indicates a pressure gradient exists between the venous territories in childhood hydrocephalus. 
In this study we have used changes in blood flow to estimate the magnitude of the pressure difference which should exist within the venous system. It has been found that an elevation in venous pressure from whatever cause, directs a larger percentage of the arterial inflow to exit via the smaller venous channels through the scalp, face and over the brain convexity through the veins of Trolard and Labbe as collateral flow. This collateral flow reduces the percentage of the arterial inflow returning via the main pathways in children at risk of idiopathic intracranial hypertension (IIH) by $11 \%$ in the SSS and $4 \%$ in the straight sinus 14 . This represents a collateral flow bypassing the main sinuses of $146 \mathrm{ml} / \mathrm{min}$ and $53 \mathrm{ml} / \mathrm{min}$ respectively. This indicates an increase in pressure both within the superficial and deep venous territories. Children with IIH require a minimum elevation in ICP of $3.8 \mathrm{mmHg}$ above normal to be symptomatic, with this all originating from causes below the Torcular ${ }^{15}$. Therefore, we can equate the $146 \mathrm{ml} / \mathrm{min}$ reduction in sagittal sinus venous return in IIH with an estimated minimum $4 \mathrm{mmHg}$ increase in sagittal sinus pressure. In the current study the superior sagittal sinus percentage venous return was reduced by $12.9 \%$ or $136 \mathrm{ml} / \mathrm{min}$, suggesting a similar $4 \mathrm{mmHg}$ increase in venous pressure. Unlike $\mathrm{IIH}$, the percentage venous return in the straight sinus deep venous territory was unchanged in hydrocephalus, suggesting the pressure change arises above the Torcular within the superficial venous territory and not the deep territory. Aso et al. using a different MRI technique came to the same conclusion, suggesting that ventriculomegally is correlated with a relative increase to the resistance to blood flow in the superficial venous system of the brain compared to the preserved drainage in the deep system ${ }^{16}$.

In a study into children with hydrocephalus, there was a $35 \%$ effective area stenosis of the sagittal sinus 7. In a modelling study, a $38 \%$ reduction in the sagittal sinus area in hydrocephalus lead to a $0.7 \mathrm{mmHg}$ increase in venous pressure ${ }^{1}$. So the effect of the stenosis in the sagittal sinus in children is probably less than $1 \mathrm{mmHg}$. This leaves a minimum $3 \mathrm{mmHg}$ of pressure elevation elsewhere in the venous system to account for the collateral flow in hydrocephalus in children. Therefore, the blood flow data suggests a pressure increase in the BCVs of the sagittal sinus of a minimum of $3 \mathrm{mmHg}$ but no increase in the vein of Galen.

In hydrocephalus, the positive correlation between the sagittal sinus outflow volume and BCV area in hydrocephalus indicates that blood flow affects the transmural pressure similar to that in dogs made hyperaemic by elevating the inspired carbon dioxide level (see Fig. 2a). In hydrocephalus there was a positive correlation between the size of the BCVs and the superior sagittal sinus percentage return (Fig. 2b), suggesting that in those individuals who have a poorer availability of collateral pathways, there are higher transmural BCV pressures. In this study there was an average $22 \%$ increase in BCV area in hydrocephalus compared to controls. The modelling performed suggests a $4.1 \mathrm{mmHg}$ increase in transmural pressure to bring this about. This modelling was based on a study comparing cadaver BCV vein size to angiography ${ }^{8}$ which was used to estimate the circumferential Young's modulus. This was necessary because the circumferential modulus has not been measured in humans. The longitudinal BCV modulus, however, has been measured and found to be $30.7 \mathrm{MPa}$ in one study ${ }^{17}$ and $25.7 \mathrm{MPa}$ in another ${ }^{9}$. The orientation of the collagen fibres in the body of the BCVs is longitudinal ${ }^{11}$ indicating the longitudinal modulus is likely to be much higher than the circumferential one. In pigs, there is a linear 
response of the diameter of the bridging veins to the transmural pressure. At zero transmural pressure the mean diameter was $0.90 \mathrm{~mm}$ and at $8 \mathrm{mmHg}$ it was $1.07 \mathrm{~mm}$ with a mean wall thickness of $0.03 \mathrm{~mm}{ }^{18}$. Thus, the circumferential modulus in pigs can be estimated using equation (1) to be $0.08 \mathrm{MPa}$ compared to our value in humans of $0.163 \mathrm{MPa}$ and both compare to the $0.4 \mathrm{MPa}$ found in silicone tubes ${ }^{19}$.

The bridging vein for the deep system is the vein of Galen. It was found to average $11.2 \mathrm{~mm}^{2}$ in controls in the present study which compares to a study using MRV measurement where the average vein of Galen was $4.04 \mathrm{~mm}$ in diameter equivalent to $12.8 \mathrm{~mm}^{2}$ in area ${ }^{20}$. There was no difference between the hydrocephalus patients and controls indicating likely no increase in transmural pressure.

The passive Starling resistor model of the BCVs is incomplete. There appears to be no anatomical reason a transmural pressure significantly higher than normal can be maintained by the venous system. In the current study all of the cortical veins in the hydrocephalus patients appeared fully open all the way to the sagittal sinus. Figure $3 c$ is an example from patient 2 who had obstructive hydrocephalus. Given there is no structural reason for an increase in transmural pressure, the question arises, could there be a functional cause?

Mahat et al. noted, vessels of differing elastic properties such as in the cardiovascular system may exhibit the characteristics of an impedance pump ${ }^{21}$. Hickerson et al. define an impedance pump as a device which uses a mismatch in impedance between two adjacent tubes to drive flow without valves ${ }^{22}$. By compressing the more compliant tube periodically in a position adjacent to the less compliant, pressure waves are set up which reflect at the points of impedance mismatch. The constructive interference of these waves will generate a net flow. The flow rate is non-linearly dependant on the compression frequency and is pulsatile. Hickerson et al. define 4 criteria to indicate if an impedance pump is operating; 1) a difference in impedance between the tubes, 2) distal compression point in the compliant tube, 3) maximal flow occurs at the resonant frequency and 4) flow exiting the tube is typically pulsatile. Clearly, there is an impedance mismatch between the cortical veins and the sagittal sinus satisfying criteria 1). With regards to criteria 2) and the site of BCV compression. The peak pulse in the cortical veins lags behind the sinuses, indicating that the pulse exits the arterial tree passing into the subarachnoid space and re-enters the cortical vessels just before their junction with the sinuses ${ }^{23}$ i.e. at the cuff region of the vein. In the deep system there is evidence of compression of the distal vein of Galen as it joins the straight sinus ${ }^{24}$. In criteria 3 ), maximum efficiency occurs at the resonant frequency of the system. In dogs, the intracranial system selectively absorbs the intracranial pulse pressure waves at the resonant cardiac frequency ${ }^{25}$. Similarly, the normal human intracranial system selectively absorbs the CSF pulse pressure at a resonant frequency around the heart rate i.e.1.49-2.05 Hz (89-123 beats per minute) ${ }^{26}$. Finally, using criteria 4), the flow exiting the cortical veins is pulsatile. Measurement of the bridging vein flow upstream from the compression site in normal patients gave a pulsatility index (P.I.) of $0.42{ }^{27}$. Therefore, we suggest the bridging cortical veins fulfil all of Hickerson et al's. criteria for being an impedance pump. How would this materially affect the transmural pressure? The highest flow rate in a $2 \mathrm{~mm}$ silicone impedance pump was $16 \mathrm{ml} / \mathrm{min}^{19}$. From our study, the sagittal sinus flow rate is 558 
$\mathrm{mls} / \mathrm{min}$ and there are on average 18 cortical veins providing this flow ${ }^{11}$, therefore each cortical vein has an average flow of $31 \mathrm{mls} / \mathrm{min}$. Therefore, impedance pumping could significantly reduce the BCV pressure if operating efficiently. Indeed, the geometry of the cortical veins may be optimised to improve pumping efficiency. It has been shown that placing a loop in an impedance pump increases the average flow rate by between $31-72 \%{ }^{28}$. The BCVs loop backwards and empty against the SSS flow with the average outflow angle being $35^{\circ} 8$. Similarly, placing a dilatation at the site of the tube compression (increasing the diameter by $20 \%$ compared to a straight tube) can increase the average flow by 4 fold ${ }^{29}$. The outflow cuff as previously described is such a dilatation.

If an efficiently performing BCV impedance pump significantly reduces the transmural pressure in normal individuals we could hypothesise that failure of such a mechanism in hydrocephalus could account for the elevated transmural pressures found. In hydrocephalic dogs, the pulsation absorber mechanism appears lost ${ }^{30}$. In hydrocephalic dogs there was a $53 \%$ increase in ventricular pulse pressure and an $85 \%$ increase in cortical vein pulse pressure but the sagittal sinus pulse pressure was not significantly changed compared to controls ${ }^{31}$, indicating pulsation absorber failure.

In human hydrocephalus, the resonant pulsation absorber mechanism is also lost. ${ }^{26}$. In the intracranial cavity the pulsation absorption consists of two components, the CSF pulsating backwards and forwards in the spinal canal and the venous compression ${ }^{23}$. In an impedance pump consisting of two compression regions in differing locations maximal flow will occur at a fixed phase lag between the two regions of compression. In an experimental system consisting of two compression regions, a fixed phase lag of $55^{\circ}$ gave the maximal flow rate. Reducing the phase lag to zero reduced the flow rate 10 fold $^{32}$. In humans the phase lag from the artery expansion to the spinal canal at C2 level is $+5.1 \pm 10.5^{\circ} 33$. The phase lag in the sagittal sinus is $-50^{\circ}$ after the arterial pulse ${ }^{27}$, giving a phase difference of $55^{\circ}$ between the spinal canal pulsation and the cortical vein compression. In hydrocephalus of middle age, this lag is reduced by $58 \%{ }^{34}$ and by $51 \%$ in elderly NPH patients ${ }^{35}$. The shift occurs because the craniospinal compliance is reduced in hydrocephalus with a significantly reduced amount of CSF expelled from the foramen magnum despite the increased CSF pulse pressure ${ }^{36}$. The alteration in phase associated with hydrocephalus would decrease the phase difference from $55^{\circ}$ to $23^{\circ}$ and significantly reduce the pumping efficiency.

In addition, increasing the outflow resistance in an impedance pump model reduced the peak flow rate because the tube was made stiffer ${ }^{22}$. Note, in dogs, increasing the outflow resistance by jugular vein compression increased the transmural venous pressure by $81 \%^{5}$. Similarly, increasing the outflow pressure by increasing the transmural pressure within the tube in an experimental impedance pump from $2.8 \mathrm{mmHg}$ to $6.6 \mathrm{mmHg}$ reduced the peak flow to zero ${ }^{22}$. These pressures are almost identical to the ones modelled in the normal and hydrocephalic children in this study. Suggesting a complete failure of impedance pumping in children may exist. This sets up a positive feedback loop, i.e. an increase in BCV transmural pressure will both be the result of, and also contribute to, the failure of the impedance pumping. Such a positive feedback loop would be expected to be unstable over short time periods and 
lead to variations in ICP over time. These pressure waves are known as B waves and are a feature of the ICP in hydrocephalus ${ }^{37}$. Occurring in both communicating and obstructive hydrocephalus ${ }^{38}$. In human hydrocephalus, the BCV outflow impedance appears to increase, with the upstream pulsatility index being 0.28 (compared to 0.42 in controls). This rebounds to 0.8 once the intracranial compliance is increased by intraventricular shunt placement. The straight sinus pulsatility was unchanged from normal both before and after shunting ${ }^{39}$, suggesting it is unaffected.

Hydrocephalus tends to be initially diagnosed either by head ultrasound in the infantile period or by CT scanning later. The MRI studies reviewed in this paper were requested by the referring neurologist or neurosurgeon at their discretion and thus there is a possible selection bias with those patients deemed to be at greater risk i.e. either symptomatic or with larger ventricles being over represented in this study. Despite this, there was no appreciable correlation between the metrics used and either the patients symptoms or the ventricular size.

\section{Methods}

\section{Subjects}

In a previous study, the radiology information system at a tertiary referral hospital was retrospectively interrogated to retrieve all data from children between birth and 15 years of age who had an MRI with MRV and flow quantification, for the investigation of any form of hydrocephalus between January 2009 and January $2019^{7}$. Eighteen children between 4 and 15 years were enrolled from this study into the current study. The selection criteria for the original study were a treatment naive hydrocephalus not secondary to tumor. The ventricular size index was measured on axial T2 images as the size of the frontal lateral ventricles compared to the size of the frontal lobes and a figure of 0.3 was used as a cutoff. Obstructive hydrocephalus was diagnosed if there was no flow through the aqueduct of Silvius and communicating hydrocephalus if the aqueduct stroke volume was above 40 microliters per systole on dedicated flow quantification imaging. There were 7 with obstructed hydrocephalus with no flow and 11 with communicating hydrocephalus with an average aqueduct flow of $201 \pm 215 \mu$ l. There were 8 females and 10 males. The pediatric neurologist (GMS) designated the patients as active or compensated hydrocephalus based on a chart review. Further information about the ventricular size and clinical findings is available in the previous study ${ }^{7}$. Seventy two control MR venogram (MRV) patients were enrolled from the previous study ${ }^{7}$. They had an MRI study for indications not related to headaches, large head, raised intracranial pressure or hydrocephalus, in which the subsequent MRI was found to be normal. There were 40 males and 32 females. There were 22 control patients with MR flow quantification studies reenrolled from the previous study with 10 males and 12 females.

\section{Ethics approval and consent to participate}

Informed consent was obtained from all patients and parents enrolled in this study. The study was approved by the Hospital ethics committee, therefore, the study has been performed in accordance with 
the ethical standards laid down in the 1964 Declaration of Helsinki. The authorization number 2019/ETH12487 was issued.

\section{MR and Analysis}

All patients were imaged on a 3.0 T superconducting magnet (Avanto; Seimens, Erlangen Germany). In all patients, a standard brain MRI consisting of 3DT1 sagittal, T2 axial, FLAIR axial and diffusion weighted axial images was performed. An MR phase contrast flow quantification sequence was acquired with retrospective cardiac gating. The TR was $26.5 \mathrm{msec}$, TE $6.9 \mathrm{msec}$, flip angle $15^{\circ}$, slice thickness $5 \mathrm{~mm}$, matrix $192 \times 512$, FOV 150 and a single excitation. The first velocity encoding value was $150 \mathrm{~cm} / \mathrm{sec}$ with the plane set to pass through the skull base, to cross the carotid and basilar arteries. A second acquisition had a velocity encoding value of $40 \mathrm{~cm} / \mathrm{sec}$ and was angled to measure the mid portion of the straight sinus and the sagittal sinus approximately $3 \mathrm{~cm}$ above the Torcular as per a previous study ${ }^{14}$. A time of flight MRV acquisition was performed in the off sagittal plane. The MRI imaging was sourced from the hospital picture archiving and communication system (PACS) and therefore all measurements were performed on the original data.

Areas of interest were placed around the carotid and basilar arteries for all patients, to give the total arterial inflow at the skull base by summing the individual flows. The arterial inflow data has been previously published ${ }^{7}$. Regions of interest were placed around the straight sinus and superior sagittal sinus to give the two venous sinus outflows. This data has not been previously published. Background subtraction was used to remove the effect of eddy currents in all results. The percentage of the arterial inflow drained by both the sagittal sinus and the straight sinus was calculated for each patient. The MRV data was reformatted to display the length of the sagittal sinus (see fig 3). The two largest BCVs on the right and left sides were selected and reconstructions performed perpendicular to their longitudinal axis 1 $\mathrm{cm}$ from their junction with the sinus (see fig 3c). The cross-sectional area was measured (see fig 3d). The four veins were then averaged for each patient. The vein of Galen was measured $5 \mathrm{~mm}$ from its junction with the straight sinus perpendicular to its longitudinal axis (see fig $3 \mathrm{e}$ ). Mean and standard deviations were obtained for each group. A Shapiro-Wilk Test was used to test for normality of the data. Differences between groups were tested using a Mann-Whitney $U$ test. Correlation amongst variables was performed using a Spearman's Rho test. An a£0.05 was used to assess statistical significance for all tests

Mathematical modelling to estimate the transmural pressure was performed. The equation relating the transmural pressure to the cross-sectional area of a vessel is

$$
P t m=\frac{4 E h}{3 R o}\left(1-\sqrt{\frac{A o}{A}}\right)
$$

Where Ptm is the transmural pressure, $\mathrm{E}$ is the circumferential Young's modulus of the wall, $\mathrm{h}$ is the wall thickness, $R o$ is the radius in the stress free state, Ao is the area in the stress free state and $A$ is the area following the applied transmural pressure ${ }^{32}$. 


\section{Declarations}

Additional Information

\section{Authors' contributions}

Conceptualisation and design GAB, ARB. Imaging data acquisition GAB. Clinical data acquisition GMS. Mathematical analysis and statistics ARB. Writing original draft GAB. Review and editing all authors. All authors read and approved the final manuscript

\section{Competing interests}

The authors declare no competing interests.

\section{Data availability}

All data generated or analysed during this study are included in this published article (and its Supplementary Information file).

\section{Funding}

There was no funding body involved in this paper.

\section{References}

1. Bateman, G. A. \& Bateman, A. R. Differences in the Calculated Transvenous Pressure Drop between Chronic Hydrocephalus and Idiopathic Intracranial Hypertension. AJNR Am J Neuroradio/ 40, 68-73, doi:10.3174/ajnr.A5883 (2019).

2. Bateman, G. A. The pathophysiology of idiopathic normal pressure hydrocephalus: cerebral ischemia or altered venous hemodynamics? AJNR Am J Neuroradiol 29, 198-203, doi:10.3174/ajnr.A0739 (2008).

3. Schaller, B. Physiology of cerebral venous blood flow: from experimental data in animals to normal function in humans. Brain Res Brain Res Rev 46, 243-260, doi:10.1016/j.brainresrev.2004.04.005 (2004).

4. Portnoy, H. D., Chopp, M., Branch, C. \& Shannon, M. B. Cerebrospinal fluid pulse waveform as an indicator of cerebral autoregulation. J Neurosurg 56, 666-678, doi:10.3171/jns.1982.56.5.0666 (1982).

5. Portnoy, H. D., Branch, C. \& Castro, M. E. The relationship of intracranial venous pressure to hydrocephalus. Childs Nerv Syst 10, 29-35, doi:10.1007/BF00313582 (1994).

6. Olivero, W. C., Rekate, H. L., Chizeck, H. J., Ko, W. \& McCormick, J. M. Relationship between intracranial and sagittal sinus pressure in normal and hydrocephalic dogs. Pediatr Neurosci 14, 196201, doi:10.1159/000120388 (1988). 
7. Bateman, G. A., Yap, S. L., Subramanian, G. M. \& Bateman, A. R. The incidence of significant venous sinus stenosis and cerebral hyperemia in childhood hydrocephalus: prognostic value with regards to differentiating active from compensated disease. Fluids Barriers CNS 17, 33, doi:10.1186/s12987020-00194-4 (2020).

8. Han, H., Tao, W. \& Zhang, M. The dural entrance of cerebral bridging veins into the superior sagittal sinus: an anatomical comparison between cadavers and digital subtraction angiography. Neuroradiology 49, 169-175, doi:10.1007/s00234-006-0175-z (2007).

9. Monea, A. G. et al. The biomechanical behaviour of the bridging vein-superior sagittal sinus complex with implications for the mechanopathology of acute subdural haematoma. J Mech Behav Biomed Mater 32, 155-165, doi:10.1016/j.jmbbm.2013.12.007 (2014).

10. Johnston, I. H. \& Rowan, J. O. Raised intracranial pressure and cerebral blood flow. 3. Venous outflow tract pressures and vascular resistances in experimental intracranial hypertension. J Neurol Neurosurg Psychiatry 37, 392-402, doi:10.1136/jnnp.37.4.392 (1974).

11. Vignes, J. R., Dagain, A., Guerin, J. \& Liguoro, D. A hypothesis of cerebral venous system regulation based on a study of the junction between the cortical bridging veins and the superior sagittal sinus. Laboratory investigation. J Neurosurg 107, 1205-1210, doi:10.3171/JNS-07/12/1205 (2007).

12. Piechnik, S. K., Czosnyka, M., Richards, H. K., Whitfield, P. C. \& Pickard, J. D. Cerebral venous blood outflow: a theoretical model based on laboratory simulation. Neurosurgery 49, 1214-1222; discussion 1222-1213, doi:10.1097/00006123-200111000-00034 (2001).

13. Chen, J. et al. Biological characteristics of the cerebral venous system and its hemodynamic response to intracranial hypertension. Chin Med J (Engl) 125, 1303-1309 (2012).

14. Bateman, G. A., Subramanian, G. M., Yap, S. L. \& Bateman, A. R. The incidence of obesity, venous sinus stenosis and cerebral hyperaemia in children referred for MRI to rule out idiopathic intracranial hypertension at a tertiary referral hospital: a 10 year review. Fluids Barriers CNS 17, 59, doi:10.1186/s12987-020-00221-4 (2020).

15. Bateman, A. R., Bateman, G. A. \& Barber, T. The relationship between cerebral blood flow and venous sinus pressure: can hyperemia induce idiopathic intracranial hypertension? Fluids Barriers CNS 18, 5, doi:10.1186/s12987-021-00239-2 (2021).

16. Aso, T. et al. A venous mechanism of ventriculomegaly shared between traumatic brain injury and normal ageing. Brain 143, 1843-1856, doi:10.1093/brain/awaa125 (2020).

17. Delye, H. et al. Biomechanical properties of the superior sagittal sinus-bridging vein complex. Stapp Car Crash J 50, 625-636 (2006).

18. Pang, Q., Lu, X., Gregersen, H., von Oettingen, G. \& Astrup, J. Biomechanical properties of porcine cerebral bridging veins with reference to the zero-stress state. J Vasc Res 38, 83-90, doi:10.1159/000051033 (2001).

19. Rinderknecht, D., Hickerson, A. I. \& Gharib, M. A valveless micro impedance pump driven by electromagnetic actuation. Journal of Micromechanics and Microengineering 15, 861-866, doi:10.1088/0960-1317/15/4/026 (2005). 
20. Deniz C. , G. E., Acu B. , Kuyucu Y. E. Comparative evaluation of dural venous sinuses and cerebral veins using contrast-enhanced spoiled gradient recalled echo and time-of-flight magnetic resonance venography. J Contemp Med 9, 214-221, doi:https://doi.org/10.16899/jcm.556044 (2019).

21. Mahat, M. M. et al. Impedance Induce Valve Pump in Closed Loop System. Applied Mechanics and Materials 393, 747-752, doi:10.4028/www.scientific.net/AMM.393.747 (2013).

22. Hickerson, A. I., Rinderknecht, D. \& Gharib, M. Experimental study of the behavior of a valveless impedance pump. Experiments in Fluids 38, 534-540, doi:10.1007/s00348-005-0946-z (2005).

23. Bateman, G. A. Pulse-wave encephalopathy: a comparative study of the hydrodynamics of leukoaraiosis and normal-pressure hydrocephalus. Neuroradiology 44, 740-748, doi:10.1007/s00234002-0812-0 (2002).

24. Bateman, G. A. Pulse wave encephalopathy: a spectrum hypothesis incorporating Alzheimer's disease, vascular dementia and normal pressure hydrocephalus. Med Hypotheses 62, 182-187, doi:10.1016/s0306-9877(03)00330-x (2004).

25. Zou, R. et al. Intracranial pressure waves: characterization of a pulsation absorber with notch filter properties using systems analysis: laboratory investigation. J Neurosurg Pediatr 2, 83-94, doi:10.3171/PED/2008/2/7/083 (2008).

26. Park, E. H., Eide, P. K., Zurakowski, D. \& Madsen, J. R. Impaired pulsation absorber mechanism in idiopathic normal pressure hydrocephalus: laboratory investigation. J Neurosurg 117, 1189-1196, doi:10.3171/2012.9.JNS121227 (2012).

27. Bateman, G. A. Cerebrospinal fluid pulsation. J Neurosurg 106, 197-199; author reply 199-202, doi:10.3171/jns.2007.106.1.197 (2007).

28. Hiermeier, F. \& Manner, J. Kinking and Torsion Can Significantly Improve the Efficiency of Valveless Pumping in Periodically Compressed Tubular Conduits. Implications for Understanding of the FormFunction Relationship of Embryonic Heart Tubes. J Cardiovasc Dev Dis 4, doi:10.3390/jcdd4040019 (2017).

29. Kozlovsky, P., Rosenfeld, M., Jaffa, A. J. \& Elad, D. Dimensionless analysis of valveless pumping in a thick-wall elastic tube: Application to the tubular embryonic heart. J Biomech 48, 1652-1661, doi:10.1016/j.jbiomech.2015.03.001 (2015).

30. Park, E. H., Dombrowski, S., Luciano, M., Zurakowski, D. \& Madsen, J. R. Alterations of pulsation absorber characteristics in experimental hydrocephalus. J Neurosurg Pediatr 6, 159-170, doi:10.3171/2010.5.PEDS09142 (2010).

31. Castro, M. E., Portnoy, H. D. \& Maesaka, J. Elevated cortical venous pressure in hydrocephalus. Neurosurgery 29, 232-238, doi:10.1097/00006123-199108000-00011 (1991).

32. Zislin, V. \& Rosenfeld, M. Impedance Pumping and Resonance in a Multi-Vessel System. Bioengineering (Basel) 5, doi:10.3390/bioengineering5030063 (2018).

33. Wagshul, M. E., Chen, J. J., Egnor, M. R., McCormack, E. J. \& Roche, P. E. Amplitude and phase of cerebrospinal fluid pulsations: experimental studies and review of the literature. J Neurosurg 104, 810-819, doi:10.3171/jns.2006.104.5.810 (2006). 
34. Bateman, G. A. Magnetic resonance imaging quantification of compliance and collateral flow in lateonset idiopathic aqueductal stenosis: venous pathophysiology revisited. J Neurosurg 107, 951-958, doi:10.3171/JNS-07/11/0951 (2007).

35. Bateman, G. A., Lechner-Scott, J. \& Lea, R. A. A comparison between the pathophysiology of multiple sclerosis and normal pressure hydrocephalus: is pulse wave encephalopathy a component of MS? Fluids Barriers CNS 13, 18, doi:10.1186/s12987-016-0041-2 (2016).

36. Katayama, S., Asari, S. \& Ohmoto, T. Quantitative measurement of normal and hydrocephalic cerebrospinal fluid flow using phase contrast cine MR imaging. Acta Med Okayama 47, 157-168, doi:10.18926/AMO/31595 (1993).

37. Kasprowicz, M., Bergsneider, M., Czosnyka, M. \& Hu, X. Association between ICP pulse waveform morphology and ICP B waves. Acta Neurochir Supp/ 114, 29-34, doi:10.1007/978-3-7091-0956-4_6 (2012).

38. Green, L. M., Wallis, T., Schuhmann, M. U. \& Jaeger, M. Intracranial pressure waveform characteristics in idiopathic normal pressure hydrocephalus and late-onset idiopathic aqueductal stenosis. Fluids Barriers CNS 18, 25, doi:10.1186/s12987-021-00259-y (2021).

39. Bateman, G. A. The reversibility of reduced cortical vein compliance in normal-pressure hydrocephalus following shunt insertion. Neuroradiology 45, 65-70, doi:10.1007/s00234-002-0901-0 (2003).

\section{Figures}

\section{Figure 1}

a A diagram of the bridging cortical vein (BCV), lateral lacunae (LL), superior sagittal sinus (SSS) and lateral ventricle (LV) with pressures in normal dogs appended from Portnoy et al. 5. The pressure differences across vein walls are shown in red with arrows. b A diagram of the pressures found in dogs with a naturally occurring form of hydrocephalus from Portnoy et al. 5 . Note the pressure across the sagittal sinus wall is increased by $1.7 \mathrm{mmHg}$ and the vein is compressed. The pressure across the BCV is increased by $327 \%$ and the vein is depicted as dilated. c A coronal T2 image of a normal dog with the arrow indicating the superior sagittal sinus. d A coronal T2 image of a naturally hydrocephalic dog showing the sagittal sinus to be much smaller than in 1c. e A sagittal T2 slice, just off centre, in a normal dog showing a BCV as a black dot at the arrow head. $f$ A sagittal slice from the hydrocephalic dog showing significantly dilated BCVs (arrows). Scatter plots of significant correlations 
Hydrocephalus SSS flow vs BCV Area

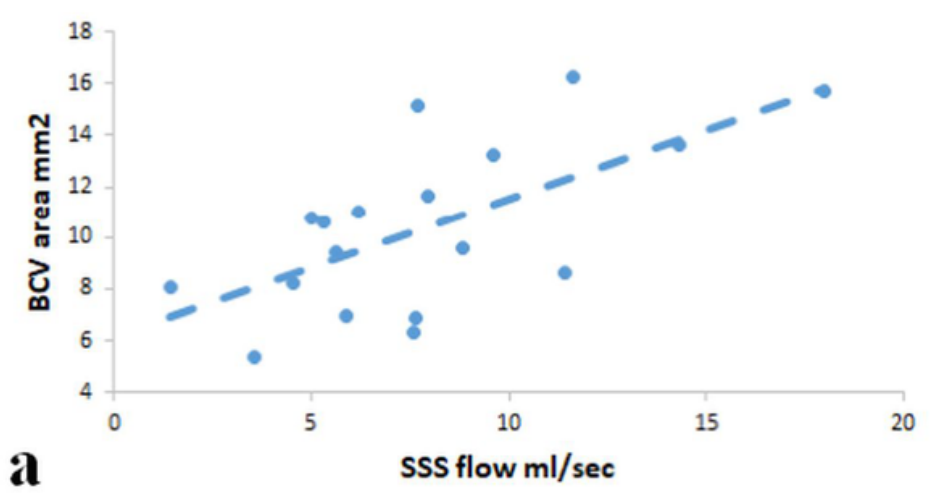

Hydrocephalus SSS \% return vs BCV Area

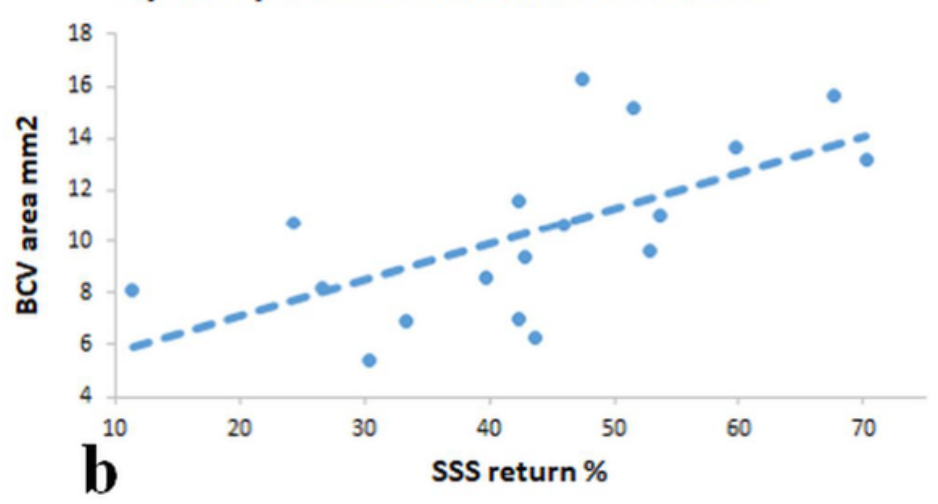

Figure 2

a A scatter plot of hydrocephalus superior sinus flow vs bridging cortical vein area showing a positive correlation $(r=0.65, p=0.004)$ b A scatter plot of hydrocephalus superior sagittal sinus percent return vs bridging cortical vein area showing a positive correlation $(r=0.67, p=0.002)$ Site of vein measurements in a child with obstructed hydrocephalus

\section{Figure 3}

a sagittal T1 image of patient 2 showing the obstructed aqueduct of Silvius (arrow). b Axial T2 image showing the ventricular dilatation. c Curved maximum intensity projection image obtained from the MRV data orientated along the course of the superior sagittal sinus (outflow towards the right) showing the site and orientation of the BCV measurements (white lines). d MRV reconstruction of a cortical vein showing an area measurement. e MRV reconstruction showing the site of the vein of Galen measurement (white line).

\section{Supplementary Files}

This is a list of supplementary files associated with this preprint. Click to download.

- corticalveindata.xlsx 